\title{
Consequences
}

$W_{\text {tition }}^{\text {he }}$ hen a small German company develops a novel biological therapy that bombs in a phase 1 trial, usually no one notices. The trial stops, the trial volunteers recover from the adverse effects, the company fails to attract any more capital and goes out of business. The event has no wider repercussions: it is merely written off as natural fallout from the need within biotech to place bets on unconventional approaches to contribute to the renewal of healthcare approaches.

The failure of TeGenero's monoclonal antibody (mAb) drug, TGN1412, in a phase 1 trial for the treatment of B-cell chronic lymphocytic leukemia has not gone unnoticed, however. TGN1412 is a 'superagonistic' mAb whose target is the immune costimulatory T-cell antigen CD28. To a cartoon approximation, it stimulates $\mathrm{T}$-cell activation directly by binding to CD28 without the need for an antigen-specific interaction. In essence, it overrides the antigen-dependent specificity of the usual costimulation process.

The attention focused on TGN1412 was guaranteed by the dramatic nature of the adverse events in the trial. Within hours of administration, all six volunteers who received the drug were suffering from multiple organ failure and had lost consciousness. A week later all were still in intensive care. As Nature Biotechnology went to press, it looked unlikely that anyone was going to die, but it was still not clear what had gone wrong. It was very clear, whatever the explanation, that the event would have some profound consequences.

Explanation 1: the wrong dose of the drug was used. Consequence: TGN1412 at the proper dose may have a future as a potential therapy, but the notion that small biotech companies can't be trusted to undertake clinical development would be reinforced.

Explanation 2: an inappropriate clinical protocol was applied. Consequence: again the naivety of those running the trial will be ques- tioned. Several commentators have suggested that volunteers in the trial should not all have been treated simultaneously, but sequentially over a period of days. The appearance of severe adverse effects in one subject would at least have spared the others. It was also suggested that localized administration of the drug — on the skin, for instance — should have preceded systemic treatment. Others have said that the phase 1 study should have involved leukemic patients whose immune response obviously needed stimulation, unlike healthy volunteers.

Explanation 3: preclinical results are not a good guide to human outcomes for biologicals. Consequence: regulators will, quite rightly, question the validity of animal studies but will probably conclude, not so correctly, that an entire swath of additional testing in different models is required for biologics. TGN1412 is described as a 'fully humanized' $\mathrm{mAb}$. From first principles it would be expected that the response to such a molecule in a nonhuman model would be different from that in a human owing partly to the immunogenicity of the molecule and partly to specificity differences in the interaction with CD28.

Explanation 4: superagonistic molecules directed against parts of a cascading immune system are not a good idea until the system is much better understood. Consequence: regulators will scrutinize very closely the underlying mechanisms of disease and companies looking to test superagonists in humans will need to put their mechanisms of action on a very solid footing.

Explanation 5: mAbs are dangerous. Consequence: investors get the yips about antibody drugs and reevaluate investments across biotech. This, of course, is not a credible explanation: mAbs per se are not dangerous. But that doesn't mean that the consequence won't ensue. Expect to see a lot of investor relations and public relations activity from companies with $\mathrm{mAbs}$ in development.

\section{Transgenic milk prospects turn sour}

The European Medicines Agency's (EMEA) decision back in February on recombinant human antithrombin- $\alpha$, an anticoagulant developed by GTC Biotherapeutics, was eagerly awaited because the product, known as Atryn, if approved, would be the first drug produced in a transgenic farm animal to reach the market. In the event, the EMEA did not approve the product, but not because of any direct concerns about its animal origins. Atryn was rejected because GTC simply did not present enough appropriate data to allay EMEA's concerns about its immunogenicity.

GTC has been developing Atryn since 1993 principally for treating patients suffering from hereditary antithrombin deficiency, a rare condition affecting one person in every 3,000-5,000 that puts them at increased risk of deep vein thrombosis. Over the years, Atryn has been given to over 200 patients. But in its submission GTC only presented data on 14 patients. This exceeds the minimum requirement of 12 laid out in the EMEA guidelines, but the authorities disallowed all but five of the cases on the basis that GTC's dosing regimen across the group of 14 had not been constant. GTC will appeal the decision. Ultimately, it may seek approval instead in the United States, where the compound is now in three phase 3 trials.

That bare-bones 'not enough data' conclusion rather skirts round some of the underlying issues that transgenic protein producers have to face.

Recombinant proteins produced in animals typically have altered glycosylation patterns compared with native proteins. This doesn't necessarily influence their pharmacological properties, of course, but in the case of Atryn, it clearly did. Compared with the conventional antithrombin- $\alpha$ product, which is extracted from bovine plasma, Atryn's serum half-life was reduced seven- to tenfold, necessitating infusion of the protein rather than a one-off injection.

But one of EMEA's principle concerns with Atryn was its potential immunogenicity. GTC claims that it has not observed adverse immunogenicity in any of the 200 patients who have received Atryn. It will be important not only for GTC but also for other animal transgenics companies to allay the concerns of regulators on this matter. The problem is that it is pretty difficult for transgenics producers to produce 'natureidentical' proteins in milk. In cows and sheep and GTC's bioreactor of choice, the goat, the oligosaccharide decoration on proteins typically contains $N$-glycolylneuraminic acid (NGNA), a monomer virtually absent in native human proteins. Furthermore, the high concentrations of protein produced in milk - around a gram per liter-stretches the glycosylation capacity of the mammary gland to its limits. In fact, only in rabbits and chickens are the oligosaccharides more human-like (containing $N$-acetylneuraminic acid).

Thus, if immunogenicity of milk-produced proteins turns out to be a generic problem, then a whole class of transgenic production methods may turn out to have a limited future. Chicken milk, anyone? 